\title{
Required for release
}

New research in the latest issue of the Journal of Virology has identified a role for Epstein-Barr virus (EBV) latent membrane protein 1 (LMP1) in the production of virus during the viral lytic cycle.

The herpesvirus EBV establishes persistent infection in humans and is associated with a broad spectrum of diseases that includes infectious mononucleosis and Burkitt's lymphoma. In in vitro culture, EBV infection of peripheral B cells creates immortalized lymphoblastoid cell lines. Three integral membrane proteins are expressed in these cell lines, including LMP1, which is essential for B-cell transformation. In the Akata cell line, in which the EBV lytic cycle can be induced, the expression of LMP1 is also observed. Ahsan et al. were interested in the biological significance of the upregulated expression of LMP1 during the lytic cycle in Akata cells.

Initially, a BAC clone containing the EBV genome from Akata cells was constructed, and the gene encoding LMP1 was disrupted by the insertion of triple stop codons. The LMP1-disrupted virus was then introduced into Akata cells, and cell clones harbouring only LMP1deleted episomes were established. Could Akata cells containing episomes with LMP1-deleted EBV produce infectious virus? Analysis of nucleocapsid formation showed that entry into lytic replication was unaffected but the infectivity of the culture supernatant was significantly reduced. It was demonstrated that this impaired infectivity was caused by a reduction in the release of virus into the supernatant, indicating that LMP1 has a crucial role in the release of virus from infected cells. This was confirmed by complementation experiments, in which expression of LMP1 in Akata cells harbouring LMP1-deleted EBV rescued virus production.

The upregulated expression of LMP1 during viral lytic replication has been known for almost 20 years, but until now its functional significance has been unclear. Here, Ahsan et al. have provided evidence that LMP1 has a crucial function in viral release during lytic infection.

$$
\text { Sheilagh Molloy }
$$

\section{(1) References and links} ORIGINAL RESEARCH PAPER Ahsan, N. et al. Epstein-Barr virus transforming protein LMP1 plays a critical role in virus production. J. Virol. 79 4415-4424 (2005)

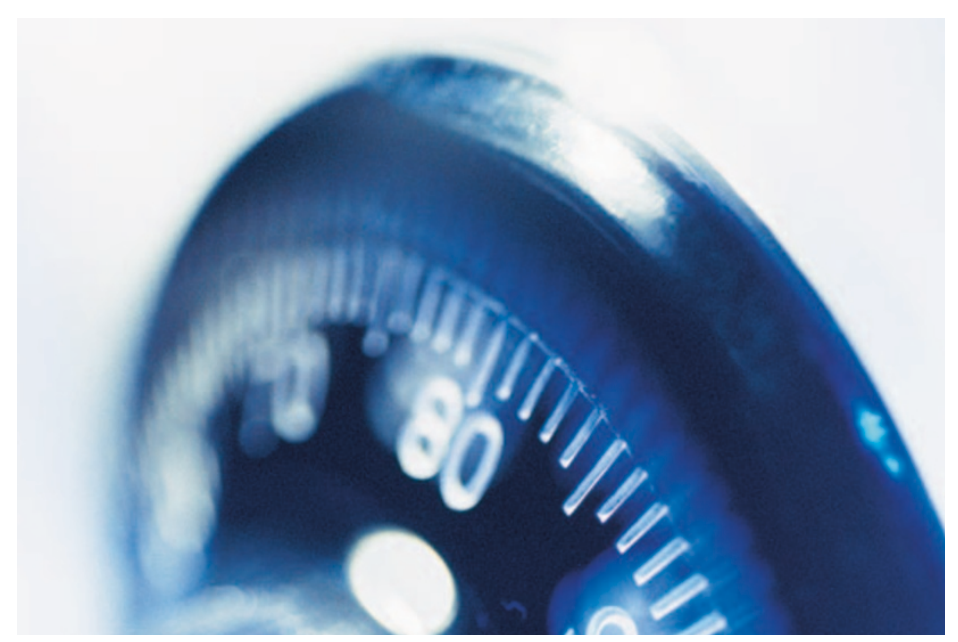

